\title{
Implications of social media misinformation on COVID-19 vaccine confidence among pregnant women in Africa
}

\author{
Farah Ennab ${ }^{\mathrm{a}, *}$, Maryam Salma Babar ${ }^{\mathrm{b}}$, Abdul Rahman Khan ${ }^{\mathrm{a}}$, Rahul Jagdishchandra Mittal ${ }^{\mathrm{c}}$, \\ Faisal A. Nawaz ${ }^{\mathrm{a}}$, Mohammad Yasir Essar ${ }^{\mathrm{d}}$, Sajjad S. Fazel ${ }^{\mathrm{e}}$ \\ ${ }^{\text {a }}$ College of Medicine, Mohammed Bin Rashid University of Medicine and Health Sciences, Dubai, United Arab Emirates \\ b Dubai Medical College, Dubai, United Arab Emirates \\ ${ }^{\mathrm{c}}$ GMERS Medical College, Gujarat, India \\ ${ }^{\mathrm{d}}$ Kabul University of Medical Sciences, Kabul, Afghanistan \\ e Department of Oncology, Cumming School of Medicine, University of Calgary, Calgary, Alberta, Canada
}

\section{A R T I C L E I N F O}

\section{Keywords}

COVID-19

Women health

Social media misinformation

Public health

Vaccine awareness

\begin{abstract}
A B S T R A C T
It has been over a year since the World Health Organization (WHO) declared the outbreak of COVID-19 as a Public Health Emergency of International Concern and subsequently a global pandemic. The world has experienced a lot of uncertainty since then as we all get used to this new 'normal' with social distancing measures, lockdowns, the emergence of new variants, and an array of hope with the development of vaccines. Having an abstract understanding of vaccine delivery, public perceptions of vaccines, and promoting acceptance of vaccines are critical to tackling the pandemic. The advent of the pandemic has led to the emergence of an 'infodemic' or rampant misinformation surrounding the virus, treatment, and vaccines. This poses a critical threat to global health as it has the potential to lead to a public health crisis by exacerbating disease spread and overwhelming healthcare systems. This 'infodemic' has led to rising vaccine hesitancy which is of paramount concern with the WHO even identifying it as one of the ten main threats to Global health almost 2 years before the approval of COVID-19 vaccines. Pregnant African women are one of the most vulnerable population groups in a region with an already burdened healthcare system. Currently, there isn't ample research in the literature that explores vaccine hesitancy in this subpopulation and the impact of social media misinformation surrounding it. The aim of this paper is to highlight the implications of this 'infodemic' on the pregnant African population and suggest key recommendations for improved healthcare strategies.
\end{abstract}

\section{Introduction}

It has been over a year since the World Health Organization (WHO) declared the outbreak of COVID-19 as a Public Health Emergency of International Concern and subsequently a global pandemic. ${ }^{1}$ The world has experienced a lot of uncertainty since then as we all get used to this new 'normal' with social distancing measures, lockdowns, the emergence of new variants, and an array of hope with the development of vaccines. Having an abstract understanding of vaccine delivery, public perceptions of vaccines, and promoting acceptance of vaccines are critical to tackling the pandemic and protecting vulnerable populations worldwide. As of October 13, 2021, there were more than 6 million confirmed cases and 148,000 reported deaths in Africa which is likely to be a profound underestimate of the actual figures due to the low testing capacity in the region. ${ }^{2-4}$

The advent of the pandemic has led to the emergence of an 'infodemic' or rampant misinformation surrounding the virus, treatment, and vaccines..$^{5-7}$ This poses a critical threat to global health as it has the potential to lead to a public health crisis by exacerbating disease spread and overwhelming healthcare systems. This 'infodemic' has led to rising vaccine hesitancy which is of paramount concern with the WHO even identifying it as one of the ten main threats to Global health almost 2 years before the approval of COVID-19 vaccines. ${ }^{8}$ Africa remains to have some of the lowest vaccination rates globally with an overall figure of only $4.4 \%$ fully vaccinated compared to $66 \%$ in the UK. ${ }^{9}$

Pregnant African women are one of the most vulnerable population

\footnotetext{
* Corresponding author.

E-mail addresses: Farah.Ennab@students.mbru.ac.ae (F. Ennab), msbsab99@gmail.com (M.S. Babar), AbdulRahman.Khan@students.mbru.ac.ae (A.R. Khan), yashmittal8866@gmail.com (R.J. Mittal), Faisal.Nawaz@students.mbru.ac.ae (F.A. Nawaz), yasir.essar@gmail.com (M.Y. Essar), sfazel3@uwo.ca (S.S. Fazel).
} 
groups in a region with an already burdened healthcare system. Currently, there isn't ample research in the literature that explores vaccine hesitancy in pregnant African women and the impact of social media misinformation surrounding it. ${ }^{10,11}$ The aim of this paper is to highlight the implications of this 'infodemic' on the pregnant African population and suggest key recommendations for improved healthcare strategies.

\section{Current misinformation on social media}

In recent years, various social media platforms are found to be riddled with unfactual statements and conspiracy theories, this abrupt wave of false medical narratives can make them an unreliable source of information for the public. Some of the false claims that have been circulated widely on social media include ingestion of sea lettuce or injecting disinfectant for protection against COVID-19. ${ }^{12,13}$

Numerous websites have claimed that Bill Gates is planning to monitor individuals and keep a digital record of their COVID-19 vaccines and testing through injected microchips, this particular claim has been shared over 3000 times on Twitter and at least 1000 times on Facebook. ${ }^{14}$ In addition, a study conducted by Jad Melki et al. showed that a large number of study subjects believe misinformation from social media platforms to be true. ${ }^{15}$

National efforts to tackle vaccine misinformation on social media in the African region.

Misinformation regarding coronavirus disease 2019 (COVID-19) poses a significant threat to public health as it has the potential to exacerbate public health issues by encouraging disease spread. Effective communication is essential to ensure that people understand how to protect themselves and others from the virus. The World Health Organization (WHO) and its partners have been working tirelessly to filter through the noise on social media in order to give reliable COVID-19 guidance. Rumors can swiftly spread online and can lead to the disruption of vaccine campaigns. In Kenya, for example, false statements regarding COVID-19 vaccines disrupting the menstrual cycle began to circulate online in May 2021.

The Africa Infodemic Response Alliance (AIRA), a WHO and partner organization program started in December 2020, used social media "listening tools" to spot misinformation and track it as it went viral. ${ }^{16} \mathrm{In}$ response to this rapidly spreading rumor, they promptly created a video featuring a well-known vaccine introduction specialist to disprove the menstrual cycle misconceptions and provide accurate information. In a matter of days, millions of people had viewed it, and is currently in the process of being translated into Kenya's native language, Kiswahili, so the Kenyan government can use it as well. ${ }^{17}$

AIRA's tools monitor social media for misleading COVID-19 claims and counter them with scientifically correct information. The technologies can identify potentially harmful rumors by counting how many people are sharing them, how quickly they spread, and how much emotion they elicit. Their primary challenge is making sure that the scientifically accurate video created becomes viral and reaches the audiences who are being misled. One of their objectives is to swiftly identify information gaps before erroneous information spreads. ${ }^{18}$

Additionally, a recent study has concluded that anti-vaccine social media users were far more effective in persuading people who were undecided about vaccines than pro-vaccine communities. ${ }^{19}$ In March, a social content hub (Viral Facts Africa) was initiated which generates and circulates content that debunks COVID-related misinformation and fills information gaps, and also works with a network of public health organizations and fact-checkers. ${ }^{20}$ The success of these initiatives is evidenced by the hundreds of millions of views on various videos and other content published by these sites.

A recent example is a story published in Nigeria's online newspaper the Whistler in June, which debunked erroneous claims concerning the COVID-19 vaccine's negative effects on pregnant women. ${ }^{21}$ Beyond COVID-19, countering disinformation is beneficial to public health.
Another collaboration was made with the UN GlobalPulse, a complementary tool that monitors talks on national, regional, and local online radios, as well as, finds influencers, and identifies rumor spreaders. These findings were shared with stakeholders so they could respond to the most widely circulated falsehoods in the region where the outbreak was occurring. ${ }^{22}$ Managing the 'infodemic' alongside the outbreak has hugely aided in bringing the crisis to an end. To date, there is insufficient information available regarding the impact of social media misinformation on vaccination hesitancy among pregnant women of various age groups in Africa.

\section{Potential implications}

With the substantial amount of misinformation that's currently being circulated via various social media platforms in the light of the COVID19 pandemic and vaccine uptake, we find that the pregnant population and notably those residing in African countries to be overwrought by this ongoing 'infodemic'. ${ }^{23}$ Pregnant women across the globe are expressing their concerns about vaccine safety and hesitancy regarding the long-term effects of the vaccine. This preconceived notion can be attributed to a few key factors; Doubt of the disease and possible implications, distrust of the healthcare system and the medical personnel, and the skepticism of vaccine efficacy during pregnancy, all of which are strongly amplified by the disseminated "outbreak" of COVID-19 misinformation on social media which seem to play a primary role in the acceptance rates of the vaccine amongst many pregnant women. ${ }^{24}$

Complications of SARS-COV-2 infection have been reported extensively in the literature, the clinical symptoms, and adverse course of infection have varied significantly amongst the different groups of the population. Some of these systemic complications affect the cardiovascular, neurological, renal, hematological, musculoskeletal, and inevitably the respiratory system. ${ }^{25}$ The overwhelming cytokine storm which has been observed in a majority of patients affected with COVID-19 has led to profound extrapulmonary complications, thus increasing the rates of hospitalization, critical care admissions, and fatality rates, ${ }^{26}$ Therefore, the urgent need for medical interventions and the enrollment of therapeutic vaccines are essential in the fight against this pandemic.

Social media has played a fundamental role in propagating false COVID-19 related vaccine narratives amongst pregnant women in Africa. This emerging wave of anti-vaccination campaigns has since created a series of apprehensive responses amongst this vulnerably unique subpopulation to vaccine confidence. ${ }^{27,28}$ As a result, this has generated profound anxiety, reluctance, fear, and hesitancy in actively seeking the COVID-19 vaccine during this critical period of time for these women. ${ }^{29}$

By paying close attention to the recent literature, we can observe some serious complications of this disease for both pregnant women and the unborn child. For instance, the incidence of preterm births, Cesarean section rates, low birth weight, and prolonged neonatal intensive care admissions appear to be higher in pregnant women affected with COVID-19 than the general population. ${ }^{30}$

Moreover, due to this ongoing health crisis, there is an increased burden on healthcare systems in Africa, adversely affecting the maternal and neonatal outcomes in a historically exhausted health system. ${ }^{31}$

Furthermore, increasing pregnancy-related complications such as preeclampsia are seen to be significantly exacerbated by the COVID-19 infection and hence leading to rapid worsening of the course of illness perinatally and postpartum. ${ }^{32}$ These possible implications as well as lessons learned from previous infections in the past during pregnancy indicate the urgent need for interventions such as accelerated vaccine rollout and reinforced vaccine uptake awareness efforts. ${ }^{33}$

\section{Recommendations for change}

Exploring the different reasons behind this prevailing hesitancy in vaccine uptake among the African pregnant population can aid in 
understanding the contributory factors and thus help maximize vaccine uptake. ${ }^{34}$ By highlighting the crucial role of social media and the impact it carries in influencing the opinions of the public, we can utilize this powerful tool in raising awareness and promoting vaccine confidence. $^{35,36}$

Various public health entities ought to work very closely alongside popular and relevant social media platforms such as WhatsApp, Facebook, Instagram, and Twitter in an integrated approach to combat vaccine misinformation. ${ }^{37}$ Conducting government surveillance programs on different social media platforms which would be protected by guidelines according to the national authorities will help in proper education and circulating evidence-based information rather than sharing myths and misconceptions surrounding the COVID-19 vaccination. ${ }^{38,39}$

Additionally, governments should dedicate adequate time to understanding and further developing effective health communication programs broadcasted in the communities and tailored to social media users as well as initiating targeted online campaigns to promote scientific awareness surrounding the COVID-19 pandemic. ${ }^{40}$

Furthermore, an online smart system can be utilized to address false and inaccurate information on social media on the basis of chatbots to help answer pregnant women's medical queries related to the COVID-19 vaccine effects and potential concerns. This innovative strategy has been employed in recent years and it has already shown promising outcomes in the virtual fight against COVID-19. ${ }^{41-43}$

We encourage and strongly recommend introducing free vaccination programs for this unique subcategory of the population as it has yielded favorable results in other countries. ${ }^{44}$ It is imperative to note the increasing psychological, emotional, and physical burden of this pandemic on pregnant women as well as the healthcare system, therefore, the introduction of such public health measures in alignment with regulated social media campaigns may tremendously result in an increased public awareness of the benefits of the vaccine during pregnancy in the African population. ${ }^{45}$

\section{Conclusion}

Misinformation related to COVID-19 has led to negative ramifications on vulnerable groups, particularly the pregnant women in the African region. This 'infodemic' adds to further challenges in promoting vaccine confidence among the pregnant population. By ensuring global collaborations, online awareness campaigns, research, and funding directed to understanding gender-focused vaccine hesitancy, the healthcare burden from COVID-19 can be ameliorated.

\section{Funding}

None.

\section{Role of authors}

All individuals who meet authorship criteria are listed as co-authors who have participated adequately in this work to take public responsibility for the generated results and content, including participation in the idea, design, writing of the manuscript, or revision. Furthermore, each author listed certifies that this material or similar material has not been and will not be submitted to or published in any other publication before its appearance in the Clinical Epidemiology and Global Health Journal.

Conceptualization: FE, FN, ME; Writing original draft: FE, FN, MB, RM, AK; Review and editing: FE, FN, ME, SF.

\section{Funding}

The authors received no financial support for this research, authorship, and/or publication of this article.

\section{Declaration of competing interest}

All authors certify that they have no affiliations with or involvement in any organization or entity with any financial interest or non-financial interest in the subject matter or materials discussed in this manuscript. All authors declare that they have no competing interests.

\section{Acknowledgments}

None.

\section{References}

1 COVID-19 Public Health Emergency of International Concern (PHEIC) Global Research and Innovation Forum. Who.Int; 2021. https://www.who.int/publications/m/it em/covid-19-public-health-emergency-of-international-concern-(pheic)-global-resea rch-and-innovation-forum. Accessed October 28, 2021.

2 Coronavirus (COVID-19). WHO | Regional Office for Africa; 2021. https://www.afro. who.int/health-topics/coronavirus-covid-19. Accessed October 28, 2021.

3 Mosi L, Sylverken A, Oyebola K, et al. Correlating WHO COVID-19 interim guideline 2020.5 and testing capacity, accuracy, and logistical challenges in Africa. Pan Afr Med J. 2021;39. https://doi.org/10.11604/pamj.2021.39.89.27522.

4 Mulu A, Bekele A, Abdissa A, et al. The challenges of COVID-19 testing in Africa: the Ethiopian experience. Pan Afr Med J. 2021;38. https://doi.org/10.11604/ pamj.2021.38.6.26902.

5 Bridgman A, Merkley E, Loewen P, et al. The causes and consequences of COVID-19 misperceptions: understanding the role of news and social media. Harvard Kennedy Sch Misinf Rev. 2020. https://doi.org/10.37016/mr-2020-028.

6 The Lancet Infectious Diseases. The COVID-19 infodemic. Lancet Infect Dis. 2020;20 (8):875. https://doi.org/10.1016/s1473-3099(20)30565-x.

7 Okereke M, Ukor N, Ngaruiya L, et al. COVID-19 misinformation and infodemic in rural africa. Am J Trop Med Hyg. 2021;104(2):453-456. https://doi.org/10.4269/ ajtmh.20-1488.

8 Wiysonge C, Ndwandwe D, Ryan J, et al. Vaccine hesitancy in the era of COVID-19: could lessons from the past help in divining the future? Hum Vaccines Immunother. 2021:1-3. https://doi.org/10.1080/21645515.2021.1893062.

9 Covid-19 Vaccinations: More than 50 Nations Have Missed a Target Set by the WHO. BBC News; 2021. https://www.bbc.com/news/56100076. Accessed October 28, 2021.

10 Hunter M, Moodley J, Moran N. Perspectives on COVID-19 vaccination for pregnant women in South Africa. Afr J Prim Health Care Fam Med. 2021;13(1). https://doi.org/ 10.4102/phcfm.v13i1.2998.

11 Murewanhema G, Dzinamarira T, Herrera H, Musuka G. COVID-19 vaccination for pregnant women in Zimbabwe: a public health challenge that needs an urgent discourse. Publ Health Pract. 2021;2, 100200. https://doi.org/10.1016/j. puhip.2021.100200.

12 feature $\mathrm{n}$. The epic battle against coronavirus misinformation and conspiracy theories. Nature.com; 2021. https://www.nature.com/articles/d41586-020-01452-z. Accessed October 28, 2021.

13 Quinn E, Fazel S, Peters C. The Instagram infodemic: cobranding of conspiracy theories, coronavirus disease 2019 and authority-questioning beliefs. Cyberpsychol, Behav Soc Netw. 2021;24(8):573-577. https://doi.org/10.1089/cyber.2020.0663.

14 Staff R. False Claim: Bill Gates Planning to Use Microchip Implants to Fight Coronavirus. U.S; 2021. https://www.reuters.com/article/uk-factcheck-coronavirus-bill-gatesmicr-idUSKBN21I3EC. Accessed October 28, 2021.

15 Melki J, Tamim H, Hadid D, Makki M, El Amine J, Hitti E. Mitigating infodemics: the relationship between news exposure and trust and belief in COVID-19 fake news and social media spreading. PLoS One. 2021;16(6), e0252830. https://doi.org/10.1371/ journal.pone.0252830.

16 Africa Infodemic Response Alliance. WHO | Regional Office for Africa; 2021. https://www.afro.who.int/aira. Accessed October 28, 2021.

17 Countering COVID-19 Misinformation in Africa | Think Global Health. Council on Foreign Relations; 2021. https://www.thinkglobalhealth.org/article/countering-co vid-19-misinformation-africa. Accessed October 28, 2021.

18 Tangcharoensathien V, Calleja N, Nguyen T, et al. Framework for managing the COVID-19 infodemic: methods and results of an online, crowdsourced WHO technical consultation. J Med Internet Res. 2020;22(6), e19659. https://doi.org/ 10.2196/19659.

19 Johnson N, Velásquez N, Restrepo N, et al. The online competition between pro- and anti-vaccination views. Nature. 2020;582(7811):230-233. https://doi.org/10.1038/ s41586-020-2281-1.

20 Newsroom A, Africa W. Viral Facts Africa initiative to combat dangerous health misinformation. Who-africa.africa-newsroom.com. https://who-africa.africa-ne wsroom.com/press/viral-facts-africa-initiative-to-combat-dangerous-health-misinfor mation?lang=en; 2021. Accessed October 28, 2021.

21 COVID-19: WHO's Advice To Pregnant Women - The Whistler Nigeria. The Whistler Nigeria; 2021. https://thewhistler.ng/covid-19-whos-advice-to-pregnant-women/. Accessed October 28, 2021.

22 UN Global Pulse - Big data for development and humanitarian action. UN Global Pulse; 2021. https://www.unglobalpulse.org/. Accessed October 28, 2021.

23 Murewanhema G. Vaccination hesitancy among women of reproductive age in resource-challenged settings: a cause for public health concern. Pan Afr Med J. 2021; 38. https://doi.org/10.11604/pamj.2021.38.336.28953. 
24 Skjefte M, Ngirbabul M, Akeju O, et al. COVID-19 vaccine acceptance among pregnant women and mothers of young children: results of a survey in 16 countries. Eur J Epidemiol. 2021;36(2):197-211. https://doi.org/10.1007/s10654-021-007286.

25 Azer S. COVID-19: pathophysiology, diagnosis, complications and investigational therapeutics. New Microbes New Infect. 2020;37, 100738. https://doi.org/10.1016/j. nmni.2020.100738.

26 Zheng K, Feng G, Liu W, Targher G, Byrne C, Zheng M. Extrapulmonary complications of COVID-19: a multisystem disease? J Med Virol. 2020;93(1): 323-335. https://doi.org/10.1002/jmv.26294.

27 Wilson S, Wiysonge C. Social media and vaccine hesitancy. BMJ Glob Health. 2020;5 (10), e004206. https://doi.org/10.1136/bmjgh-2020-004206.

28 Otieno N, Otiato F, Nyawanda B, et al. Drivers and barriers of vaccine acceptance among pregnant women in Kenya. Hum Vaccines Immunother. 2020;16(10): 2429-2437. https://doi.org/10.1080/21645515.2020.1723364.

29 Murewanhema G, Dzinamarira T, Herrera H, Musuka G. COVID-19 vaccination for pregnant women in Zimbabwe: a public health challenge that needs an urgent discourse. Publ Health Pract. 2021;2, 100200. https://doi.org/10.1016/j. puhip.2021.100200.

30 Smith V, Seo D, Warty R, et al. Maternal and neonatal outcomes associated with COVID-19 infection: a systematic review. PLoS One. 2020;15(6), e0234187. https:// doi.org/10.1371/journal.pone.0234187.

31 Kimani R, Maina R, Shumba C, Shaibu S. Maternal and newborn care during the COVID-19 pandemic in Kenya: re-contextualising the community midwifery model. Hum Resour Health. 2020;18(1). https://doi.org/10.1186/s12960-020-00518-3.

32 Narang K, Enninga E, Gunaratne M, et al. SARS-CoV-2 infection and COVID-19 during pregnancy: a multidisciplinary review. Mayo Clin Proc. 2020;95(8): 1750-1765. https://doi.org/10.1016/j.mayocp.2020.05.011.

33 Schwartz D, Graham A. Potential maternal and infant outcomes from coronavirus 2019-nCoV (SARS-CoV-2) infecting pregnant women: lessons from SARS, MERS, and other human coronavirus infections. Viruses. 2020;12(2):194. https://doi.org/ 10.3390/v12020194.

34 Cooper S, van Rooyen H, Wiysonge C. COVID-19 vaccine hesitancy in South Africa: how can we maximize uptake of COVID-19 vaccines? Expert Rev Vaccines. 2021;20 (8):921-933. https://doi.org/10.1080/14760584.2021.1949291.
35 Acheampong T, Akorsikumah E, Osae-Kwapong J, Khalid M, Appiah A, Amuasi J. Examining vaccine hesitancy in Sub-Saharan africa: a survey of the knowledge and attitudes among adults to receive COVID-19 vaccines in Ghana. Vaccines. 2021;9(8): 814. https://doi.org/10.3390/vaccines9080814.

36 Meleo-Erwin Z, Basch C, MacLean S, Scheibner C, Cadorett V. To each his own": discussions of vaccine decision-making in top parenting blogs. Hum Vaccines Immunother. 2017;13(8):1895-1901. https://doi.org/10.1080/ 21645515.2017.1321182

37 Sallam M. COVID-19 vaccine hesitancy worldwide: a concise systematic review of vaccine acceptance rates. Vaccines. 2021;9(2):160. https://doi.org/10.3390/ vaccines9020160.

38 Puri N, Coomes E, Haghbayan H, Gunaratne K. Social media and vaccine hesitancy: new updates for the era of COVID-19 and globalized infectious diseases. Hum Vaccines Immunother. 2020;16(11):2586-2593. https://doi.org/10.1080/ 21645515.2020.1780846.

39 Wilson K, Atkinson K, Deeks S. Opportunities for utilizing new technologies to increase vaccine confidence. Expert Rev Vaccines. 2014;13(8):969-977. https://doi. org/10.1586/14760584.2014.928208.

40 Glanz J, Wagner N, Narwaney K, et al. Web-based social media intervention to increase vaccine acceptance: a randomized controlled trial. Pediatrics. 2017;140(6), e20171117. https://doi.org/10.1542/peds.2017-1117.

41 Almalki M, Azeez F. Health chatbots for fighting COVID-19: a scoping review. Acta Inf Med. 2020;28(4):241. https://doi.org/10.5455/aim.2020.28.241-247.

42 Dennis A, Kim A, Rahimi M, Ayabakan S. User reactions to COVID-19 screening chatbots from reputable providers. J Am Med Inf Assoc. 2020;27(11):1727-1731. https://doi.org/10.1093/jamia/ocaa167.

43 Tudor Car L, Dhinagaran D, Kyaw B, et al. Conversational agents in health care: scoping review and conceptual analysis. J Med Internet Res. 2020;22(8), e17158. https://doi.org/10.2196/17158.

44 Suliman D, Nawaz F, Mohanan P, et al. UAE efforts in promoting COVID-19 vaccination and building vaccine confidence. Vaccine. 2021;39(43):6341-6345. https://doi.org/10.1016/j.vaccine.2021.09.015.

45 Chou W, Budenz A. Considering emotion in COVID-19 vaccine communication: addressing vaccine hesitancy and fostering vaccine confidence. Health Commun. 2020;35(14):1718-1722. https://doi.org/10.1080/10410236.2020.1838096. 